\title{
Importância do enfermeiro para o controle do câncer de mama: revisão narrativa
}

\author{
Importance of the nurse for the control of breast cancer: narrative review
}

Importancia de las enfermeras para el control del cáncer de seno: revisión narrativa

Josiane Ramos Garcia Rodrigues ${ }^{1 *}$, Ariana Aparecida Lins Aleksandrovic Salun1, Vanessa Aparecida Sanches Campassi de Oliveira², Priscila Bocchile de Lima', Maria Renata Nunes'.

\section{RESUMO}

Objetivo: O presente estudo buscou através da revisão bibliográfica verificar o papel do enfermeiro na orientação do autoexame das mamas. Revisão Bibliográfica: O câncer de mama é considerado um grande problema de saúde pública, tornando imprescindível o papel do enfermeiro de educador para diminuir os casos da doença descobertos de forma tardia. Estudos realizados mostram a importância da prática do profissional de enfermagem relacionado a orientação e educação para um cuidado preventivo, tornando-o imprescindível para colocar em prática as estratégias e diagnosticar a doença precocemente, diminuindo assim casos de câncer que são descobertos tardiamente. Levando-se em conta o papel essencial do enfermeiro na prevenção e controle desta enfermidade, percebemos que suas condutas vão desde a realização da consulta de enfermagem e orientação de seus pacientes de exames necessários e participação em ações educativas, exercendo assim, além de um papel preventivo, um aliado no diagnóstico precoce da patologia. Considerações Finais: Foi possível concluir que o enfermeiro é profissional responsável para ação do controle de câncer de mama, porém apresenta dificuldades para exercer sua função sendo necessário preparo através de capacitações e conscientizações para detecção precoce do câncer de mama.

Palavras-chave: Educação em saúde, Saúde da mulher, Neoplasias da mama, Prevenção primária, Educação em enfermagem.

\begin{abstract}
Objective: The present study sought through the bibliographic review to verify the role of nurses in guiding breast self-examination. Bibliographic Review: Breast cancer is considered a major public health problem, making the role of nurse educators essential to reduce the cases of the disease discovered late. Studies carried out show the importance of nursing professional practice related to guidance and education for preventive care, making it essential to put strategies into practice and diagnose the disease early, thus reducing cases of cancer that are discovered late. Taking into account the essential role of nurses in the prevention and control of this disease, we realize that their conduct ranges from carrying out the nursing consultation and guiding their patients to the necessary exams and participation in educational actions, thus exercising, in addition to a role preventive, an ally in the early diagnosis of pathology. Final considerations: It was possible to conclude that the nurse is a professional responsible for the action of breast cancer control, but has difficulties to exercise his function, requiring preparation through training and awareness for early detection of breast cancer.
\end{abstract}

Keywords: Health education, Women's health, Breast neoplasms, Primary prevention, Education nursing.

${ }^{1}$ Faculdade de Medicina de Marília (FAMEMA). Marília - São Paulo.

*E-mail: lejorodrigues@yahoo.com.br

${ }^{2}$ Associação Feminina Maternidade e Gota de Leite. Marília - São Paulo. 


\section{RESUMEN}

Objetivo: Este estudio buscó, a través de una revisión de la literatura, verificar el papel de las enfermeras en la guía del autoexamen de los senos. Revisión bibliográfica: el cáncer de mama se considera un importante problema de salud pública, por lo que el papel de los educadores de enfermería es esencial en la reducción tardía de los casos de la enfermedad. Los estudios realizados muestran la importancia de la práctica profesional de enfermería relacionada con la orientación y la educación para la atención preventiva, por lo que es esencial implementar estrategias y diagnóstico temprano de la enfermedad, reduciendo los casos de cáncer descubiertos tarde. Teniendo en cuenta el papel esencial de las enfermeras en la prevención y el control de esta enfermedad, nos damos cuenta de que su conducta varía desde la realización de la consulta de enfermería y la orientación del paciente hasta los exámenes necesarios y la participación en acciones educativas, ejercicio, además de la prevención., un aliado en el diagnóstico precoz de la patología. Consideraciones finales: fue posible concluir que la enfermera es un profesional responsable de la acción del control del cáncer de seno, pero que tiene dificultades para realizar su función, que requiere preparación a través del entrenamiento y la conciencia para la detección temprana del cáncer de seno.

Palabras clave: Educación en salud, Salud de la mujer, Neoplasias de la mama, Prevención primaria, Educación en enfermería.

\section{INTRODUÇÃO}

O câncer de mama hoje é considerado um grande problema de saúde pública em todo o mundo, é o segundo tipo de neoplasia mais afetado nas mulheres perdendo apenas para o melanoma. É considerada uma doença temida entre as mulheres por acometer um órgão que identifica a feminilidade e a sexualidade. Apresenta uma incidência considerável a partir dos 40 anos e um aumento de até 10 vezes acima de 60 anos. Os homens também podem ser acometidos com uma proporção de $1 \%$ do total de casos da doença (BRASIL, 2020 e INCA, 2020).

A cura depende muito do tipo de tumor e do estágio em que se encontra a doença pois aqueles que são diagnosticados precocemente tem um resultado satisfatório ou proporciona melhores condições de sobrevida, por este motivo existe a necessidade de um controle para realizar exames sempre que necessário ou anualmente para identificar essas alterações iniciais proporcionando chances maiores de cura (INCA, 2020; VEIT MT E CARVALHO VA, 2010).

O controle do câncer de mama tem sido eficaz quando a doença é descoberta precocemente, possibilitando o recurso terapêutico com mais chances de cura. Consideramos que $80 \%$ das descobertas são através do autoexame das mamas, mas esta estratégia não tem sido eficiente quando detectada, pois geralmente apresentam em fase avançada. Os métodos mais seguros e eficazes são o exame clínico e mamografia (SILVA PA e RIUL SS, 2011).

Os métodos para a detecção precoce do câncer de mama como o diagnóstico e rastreamento favorecem para a redução da apresentação do câncer destacando a relevância da conscientização das mulheres e dos profissionais da saúde para o reconhecimento dos sinais e sintomas da doença, viabilizando o acesso momentâneo dos serviços de saúde (INCA, 2018).

A equipe de enfermagem tem papel fundamental na prevenção pois são eles que orientam os pacientes na prevenção primária relacionado aos melhores hábitos de vida. O enfermeiro tem dever de educador, principalmente no cenário da atenção primária onde possui capacitação e autonomia para realizar campanhas, palestras e solicitar exames e medicar devido o respaldo dos protocolos institucionais existentes (COREN, 2018). Em um estudo realizado sobre o câncer de mama, mostra a importância da prática do profissional de enfermagem relacionado a orientação e educação para um cuidado preventivo. O papel do enfermeiro torna-se imprescindível para colocar em prática as estratégias para diagnosticar a doença precocemente, diminuindo assim casos de câncer que são descobertos de forma tardia (RODRIGUES FB, et al., 2012).

REAS/EJCH | Vol.Sup.n.55 | e3668 | DOI: https://doi.org/10.25248/reas.e3668.2020 Página 2 de 9 
O autoexame de mama tem sido incentivado pelas campanhas realizadas pelos profissionais da saúde, apresentam objetivo de educar e conscientizar as mulheres sobre a importância de realizarem o autoexame das mamas afim de detectarem sinais e sintomas que possam diagnosticar precocemente o câncer de mama (ENDRIGO J e TRALDI MC, 2017).

É de extrema importância a realização do autoexame de mama e exame clínico pois eles fazem parte das medidas de rastreamento precoce do câncer de mama, através destes que podemos detectar ou encontrar sinais e sintomas para um diagnóstico precoce (ANDRADE SAF, 2014)

Acredita-se que o tema seja de grande relevância para a área da oncologia, tendo em vista o interesse de poder atuar na prevenção do câncer de mama através de estratégias de educação. Diante desse assunto, objetiva-se verificar o papel do enfermeiro na orientação do autoexame das mamas.

\section{REVISÃO BIBLIOGRÁFICA}

\section{Fisiopatologia do câncer}

O câncer é considerado como sendo um crescimento desordenado de células que invadem os tecidos e órgãos rapidamente, incontroláveis e agressivos, causando a formação de tumores malignos que podem acometer todas regiões do corpo (INCA, 2019).

O crescimento de células cancerosas é diferente do crescimento das células normais, a mesma ao invés de morrerem, continuam crescendo descontroladamente e formando outras novas células anormais. As neoplasias podem ser classificadas em benignas quando seu crescimento é de forma organizada, lenta, expansivo e apresenta limites bem nítidos, e as malignas que são capazes de invadir tecidos vizinhos e provocar metástase podendo levar a morte (INCA, 2019).

\section{Dados epidemiológicos do câncer}

A origem do câncer pode ser considerada em torno de $80-90 \%$ por fatores externos que estão presentes no meio ambiente: hábito de vida, alimentação, medicamentos e produtos químicos, as demais causas são fatores internos como condições genéticas, hereditárias, hormonais, envelhecimento e problemas imunológicos (INCA, 2019)

As estimativas do triênio 2020/2022 mostra a ocorrência de cerca de 625 mil casos novos de câncer sendo o mais frequentes no homem o câncer de próstata e nas mulheres o de mama, estes apresentam os maiores índices da doença em todas as regiões geográficas do país com exclusão da Região Norte que mostram o câncer de mama e colo de útero com resultados estatísticos equivalentes (BRASIL, 2019).

Segundo os dados da Organização Mundial da Saúde (OMS), em 2040 há uma estimativa de 29 milhões de habitantes no mundo diagnosticados com câncer, no Brasil este número está em torno de 998 mil habitantes e de 476 mil óbitos (ANHP, 2018).

\section{Tratamento do câncer}

Atualmente todas as modalidades de tratamento: cirurgia, radioterapia, quimioterapia e transplante de medula óssea são oferecidos pelo SUS (Sistema Único de Saúde) de forma gratuita e integral, sendo optado pelo médico a forma de tratamento que deve ser realizado de acordo com o tipo e estágio da doença (BRASIL, 2013). São eles:

- Cirúrgico: tem a finalidade de avaliar o estadiamento da doença e realizar a retirada do tumor totalmente com o objetivo curativo ou de forma paliativa proporcionando uma melhor qualidade de vida (INCA, 2019);

- Radioterapia: tratamento de radiação indicado para destruir ou diminuir o tumor, pode ser realizado simultâneamente com a quimioterapia (INCA, 2019);

- Quimioterapia: medicação que tem por finalidade destruir as células cancerígenas evitando sua proliferação, pode ser administrada pelas vias: tópica, oral, subcutânea, intramuscular, intravenosa e intratecal (INCA, 2019); 
- Transplante de medula óssea: indicado para algumas doenças no sangue com o objetivo de reconstruir uma medula saudável. Pode ser autogênico (dele próprio) ou alogênico (de um doador) (INCA, 2019);

- Cuidados paliativos: são cuidados prestados ao paciente e seus familiares com qualidade para diminuir o sofrimento físico, psicológico, social e espiritual daquele que apresenta uma doença que não pode ser mais curada (INCA, 2019; ONCOGUIA, 2020b).

\section{Câncer de mama: Definição}

O câncer de mama é um tumor maligno que se desenvolve através da multiplicação descontrolada das células da mama, estas apresentam diferentes formas de evolução (INCA, 2020).

É considerado como sendo uma doença bastante assustadora pelas mulheres devido a sua periocidade, recidiva, dor e associação com a morte, seus efeitos psicológicos e sua própria imagem corporal devido ao risco de mutilação podem causar dificuldades no aceitamento da patologia provocando mudanças irreversíveis em sua vida com o diagnóstico constatado (FURTADO SB, et al., 2009).

\section{Epidemiologia}

O câncer de mama no mundo é considerado a $5^{\mathrm{a}}$ causa de morte e a mais frequente entre as mulheres (WORLD HEALTH ORGANIZATION, 2020). No Brasil é o segundo tipo de câncer mais frequente depois do de pele não melanoma e a estimativa para casos novos no país para o ano de 2020 é de aproximadamente 66.280 (INCA, 2020). Nos Estados Unidos a estimativa para o mesmo ano é de 276.480 novos casos de câncer de mama invasivo e 48.530 não invasivo (AMERICAN CANCER SOCIETY, 2020). No ano de 2018 os países que apresentaram maior incidência da doença foram Austrália, Nova Zelândia, Norte da Europa e na Europa Ocidental (ONCOGUIA, 2020a).

\section{Sinais e sintomas}

Os cânceres de mama se localizam geralmente no quadrante superior externo, normalmente as lesões são fixas, indolores, e com bordas irregulares, acompanhadas de alterações da pele como abaulamentos ou retrações principalmente no mamilo e secreção papilar quando em estágio avançado. Os principais sinais e sintomas de câncer de mama são nódulos na mama e ou axila, dor mamária e alterações da pele que recobre a mama, como abaulamentos ou retrações com aspecto semelhante à casca de laranja (SILVA PA e RIUL SS, 2011).

\section{Etiologia e fatores de risco}

É uma doença multifatorial, e os fatores genéticos e ambientais contribuem para a sua ocorrência. Existem alguns fatores adicionais que aumentam os riscos como exemplo: fatores nutricionais, aspectos endócrinos como menarca precoce e menopausa tardia, idade avançada, história familiar e pessoal que incluem parentes de primeiro grau com câncer de mama antes dos 50 anos, primeira gestação após 30 anos e nuliparidade, histórico e duração da amamentação, obesidade na pós menopausa, tabagismo, consumo de álcool, exposição à radiação ionizante e nível socioeconômico (SILVA PA e RIUL SS, 2011; PROLLA CMD, et al., 2015).

\section{Prognóstico}

O câncer de mama no Brasil mesmo quando descoberto e tratado a tempo, ainda apresenta uma alta taxa de mortalidade, associado a seu diagnóstico em estágio avançado devido as dificuldades de acesso a descoberta da doença e tratamento das mulheres que dependem do SUS (PROLLA CMD, et al., 2015).

O prognóstico provém do seu estadiamento e sua representação, se o diagnóstico for realizado no início à possibilidade de cura é maior, porém se for tardiamente apresenta riscos de metástase com uma chance de sobrevida menor. Mesmo diante desta realidade, existem muitas opções de tratamento que podem proporcionar ao cliente uma melhor qualidade de vida e possibilidade de prolongamento na sua existência (INCA, 2020). As estatísticas mostram uma sobrevida relativa em 5 anos, muitas vivem mais e outras são curadas dependendo do estágio que se encontram. Existem alguns fatores que podem prejudicar 0 prognóstico do paciente como idade, estado geral e a resposta do tratamento (ONCOGUIA, 2017). 


\section{Diagnóstico}

A investigação diagnóstica de câncer de mama se inicia geralmente através de uma queixa da paciente ou quando o médico realiza um exame físico e detecta a presença de um nódulo. Após este achado é necessário realizar exames complementares como: mamografia, ultrassom, ressonância magnética e biópsia para finalmente ser realizado o diagnóstico (INCA, 2020). Segundo dados internacionais foi comprovado que ao longo do tempo da vida pelo menos uma mulher entre oito, serão diagnosticadas com câncer de mama. $O$ maior objetivo da detecção precoce do câncer de mama é a eficácia do tratamento pois quando este é diagnosticado no início ou no órgão de origem esta possibilidade de cura aumenta. Existem 2 componentes principais nos programas de detecção precoce para o câncer que são: diagnósticos precoces e rastreamento (STEIN AT, et al., 2009).

\section{Métodos de detecção precoce}

\section{- $\quad$ Auto-exame de mama}

É um dos métodos considerado mais importante para a detecção de câncer de mama, sem custo e eficaz na qual necessita apenas de um processo educativo de um profissional responsável para ensinar a técnica corretamente e detectar alterações mamárias. Através da palpação e visualização, sendo de extrema importância a mesma conhecer o seu corpo para realizar, sendo orientada realizar uma semana após a menstruação. O médico também auxilia suas pacientes a reconhecer anormalidades existentes (STEIN AT, et al., 2009).

\section{- Exame clínico da mama (ECM)}

Este exame deve ser realizado por um profissional da saúde capacitado, e só pode ser detectado alguma anormalidade dependendo do tamanho do tumor. A sensibilidade do ECM é cerca de $88 \%$ para lesões maiores que $1 \mathrm{~cm}$, mas somente $34 \%$ a $55 \%$ em tumores menores que $1 \mathrm{~cm}$. Em relação ao rastreamento do câncer de mama, o exame físico deve ser realizado anualmente a partir dos 40 anos porém o Instituto Nacional de Câncer (INCA) recomenda que o exame clínico deve fazer parte do atendimento integral à saúde da mulher em todas as consultas independente da faixa etária (STEIN AT, et al., 2009; INCA, 2018).

\section{- Inspeção}

A inspeção é importante para observar quaisquer alterações das mamas como assimetria, abaulamentos, retrações, edemas e modificações das aréolas como tamanho, forma, simetria, coloração ou descamações e dos mamilos deve estar atento a direção de apontamento bem como achatamento ou inversão. O exame deve ser realizado em pé ou sentado, primeiramente com os membros superiores pendendes do lado de seu corpo, depois elevados e por último com as mãos na cintura realizando uma leve compressão. (STEIN AT et al., 2009; BRASIL, 2001).

\section{- Palpação}

A palpação acontece em dois momentos. Primeiro com a paciente sentada deve ser realizado a palpação das axilas e regiões supra e infraclaviculares, o examinador deve apoiar o antebraço do paciente para que os músculos fiquem relaxados, os dedos do examinador deve ser posicionado da parte superior da axila e movimentado em 4 direções (face costal, umeral, peitoral e dorsal) com objetivo de detectar linfonodos bem como seu tamanho, consistência e mobilidade. $\mathrm{Na} 2^{\mathrm{a}}$ etapa é realizado a palpação do tecido mamário com a paciente deitada e com os braços elevados, inicia-se o exame com as polpas digitais com movimentos circulares do mamilo para as extremidades em sentido horário e em todo quadrante mamário avaliando a consistência, hipersensibilidade e presença de nódulos. Após esta etapa deve-se realizar a expressão suave dos mamilos para avaliar presença de secreções. (STEIN AT, et al., 2009; BRASIL, 2001).

\section{- Mamografia}

Para avaliação e diagnóstico do câncer de mama o principal método utilizado é a mamografia que está indicada a partir dos 40 anos. Programas periódicos do exame reduzem a mortalidade por câncer de mama de 25 a 32\% (MARQUES EF, et al., 2011). Segundo Stein AT, et al. (2009), o exame pode identificar o câncer 
pelo menos 2 anos antes de atingir seu tamanho detectável através da palpação.

\section{- Ultrassonografia}

A ultrassonografia também é uma avaliação importante para a detecção do câncer de mama, sendo capaz de identificar nódulos pequenos e de elucidar lesões inconclusivas na mamografia. Porém, a principal desvantagem desse método é ser altamente dependente do operador (MARQUES EF, et al., 2011).

\section{- Ressonância magnética (RM)}

Permite o estudo da vascularização das lesões através de contraste infundido no acesso venoso. A RM apresenta maior sensibilidade (94\% a 99\%), porém apresenta grande variação da especificidade (37\% a 86\%) nos estudos publicados podendo necessitar de investigação adicional (MARQUES EF, et al., 2011).

\section{- Biópsia}

É um procedimento indicado para diagnosticar a doença, este é realizado través da retirada de uma porção pequena de tecido para avaliação de um patologista que detecta o tipo de câncer. A biópsia pode ser realizada através de aspiração ou cirurgia (ONCOGUIA, 2017).

\section{Tratamento}

Com a tecnologia o tratamento apresenta diversas formas terapêuticas na qual depende do estágio que a doença se encontra, dentre esses tipos de tratamento podemos citar: cirurgia, radioterapia, quimioterapia, hormonioterapia e terapia biológica (INCA, 2020). A imagem da mulher é alterada quando a mesma necessita realizar mastectomia afetando a feminilidade e sexualidade, pois a mama é considerada um símbolo de identidade feminina, este é comparado como uma castração ou associado com uma perda de algo importante e insubstituível, devido a isso as demais opções terapêuticas, quimioterapia e radioterapia são escolhas menos angustiantes (FURTADO SB, et al., 2009).

Os profissionais, enfermeiros reconhecem a importância dos diversos tratamentos do câncer, porém as prioridades não devem recair apenas no manejo da doença, mas se estender ao ambiente construído ao seu redor (FURTADO SB, et al., 2009).

\section{Ações preventivas e educativas}

O Outubro Rosa surgiu nos Estados Unidos em 1990 através de uma fundação que lançou o laço cor-derosa que foi distribuído na $1^{\underline{a}}$ corrida pela cura do câncer de mama. Ao decorrer do século outras entidades passaram a realizar ações de prevenções, resultando pelo Congresso Norte Americano transformar oficialmente, o outubro mês de prevenção do câncer de mama (CENTRO DE INTEGRAÇÃO EMPRESAESCOLA, 2018). O INCA iniciou sua participação no Outubro Rosa em 2010 através de eventos técnicos, debates, apresentações sobre o tema além de produções de materiais e recursos educativos para disseminar informações sobre prevenção e detecção precoce (INCA, 2019).

O protocolo para detecção do câncer de mama no Brasil é recomendado pelo Ministério da Saúde (MS), o exame clínico anual para mulheres assintomáticas entre 40 e 50 anos a mamografia bianual para mulheres entre 50 e 69 anos e para mulheres com risco de desenvolver a doença já é indicado realizar anualmente com idade superior a 35 anos (PROLLA CMD, et al., 2015).

O MS recomenda a implementação de estratégias de informações para o diagnóstico precoce do câncer de mama através de campanhas. Estas devem envolver conscientização do próprio corpo e conhecimento sobre sinais e sintomas. Para sucesso nestas estratégias os profissionais da saúde devem estar capacitados para realizar ações educativas e uma estruturação de rede de atenção preparada para acolher as mulheres com suspeita do câncer de mama e garantir com qualidade toda investigação necessária para confirmação diagnóstica (INCA, 2015).

O SUS tem realizado estratégias em todos os níveis de saúde para redução de obstáculos no acesso aos serviços de saúde com objetivo de detecção precoce do câncer de mama, as informações devem ser objetivas e claras para toda a população (INCA, 2020). 
Com o estudo realizado sobre formas de prevenções primária do câncer de mama foi possível detectar que a amamentação e um estilo de vida saudável incluindo a prática regular de atividade física, consumo de álcool moderado ou ausente e manutenção de peso são fatores favoráveis para evitar ou diminuir os riscos da doença (INUMARU LE, et al., 2011; INCA, 2020).

\section{Papel do enfermeiro na prevenção do câncer de mama}

Levando-se em conta o papel essencial do enfermeiro na prevenção e controle desta enfermidade, percebemos que suas condutas vão desde a realização da consulta de enfermagem e orientação de seus pacientes de exames necessários e participação em ações educativas, exercendo assim, além de um papel preventivo, um aliado no diagnóstico precoce da patologia (CUNHA AR, et al., 2018).

Uma das barreiras apresentadas para a não realização dos exames periódicos são as condições socioeconômicas da população que dificultam o acesso ao SUS, diante disso as ações educativas do enfermeiro devem requerer métodos de comunicação com questionamentos para atingir todas as classes sociais (ENDRIGO J e TRALDI MC,2017; OHL ICB, et al., 2016).

A educação para as mulheres da realização do autoexame das mamas faz se necessário para o alcance do sucesso na luta contra o câncer, muitas mulheres perdem a oportunidade de detecção e tratamento devido à falta de informação, conhecimento e conscientização sobre a possibilidade de um bom prognóstico (SOARES CB, 2011).

A sistematização da assistência de enfermagem é importante no processo de educação e conscientização da população para promover o autocuidado na detecção precoce e diminuição dos casos de câncer de mama (PEREIRA ACA, et al., 2018). Segundo Cunha AR, et al. (2018), o enfermeiro durante a consulta de enfermagem não executa todos os passos necessários durante o exame clínico das mamas devido negligência de algumas etapas e insuficiência de conhecimentos.Em um estudo realizado mostra a importância de os enfermeiros serem capacitados para identificar possíveis sinais e sintomas precocemente tornando uma possibilidade maior de cura. ${ }^{26}$

A educação permanente nas instituições e capacitações em cursos de graduações são necessárias para que os enfermeiros desenvolvam ações educativas para o rastreamento e diagnóstico precoce do câncer de mama no Brasil (CAVALCANTE SAM, et al., 2013). Segundo Avelar MO, et al. (2014), o enfermeiro deve atuar no rastreamento, divulgação e execução do processo de educador, informando da importância da adesão do autoexame da mama, mamografia e exame clínico. Deve atuar em todos os níveis de atenção: primária, secundária ou terciária tendo a participação fundamental no processo educativo para a saúde.

Os enfermeiros devem estar capacitados e trabalhar com uma equipe multidisciplinar para enfrentar esse desafio, além de humanizar e melhorar o atendimento à mulher. É necessário que durante a consulta de enfermagem esse profissional transmita informações adequadas sobre a importância da alimentação saudável, prática de atividade física, autoexame para conhecimento do corpo e do exame clínico com periodicidade, importância para realização da mamografia assim como a periodicidade recomendada para 0 rastreamento, mesmo sem achados clínicos significativos (LEÃO MRC, et al., 2011).

De acordo com alguns estudos realizados evidenciou-se que os enfermeiros da atenção primária apresentam dificuldades de realizar integralmente a sua função de orientador para a prevenção do câncer de mama de acordo com as competências impostas pelo MS, justifica-se pela alta demanda de atendimento com sobrecarga de serviço, falta de recursos humanos e materiais, falta de conhecimento teórico e técnico e a falta de capacitação, implicando um déficit de qualidade do cuidado proporcionando as mulheres uma diminuição da chance de detectarem o câncer de mama precocemente (CUNHA AR, et al., 2018; TEIXEIRA MS, et al., 2017; OLIVEIRA MM, et al., 2017).

\section{CONSIDERAÇÕES FINAIS}

Por meio da realização deste trabalho foi possível observar de como é necessário à conscientização dos serviços de saúde para programar ações e estratégias específicas à saúde da mulher. O conhecimento que

REAS/EJCH | Vol.Sup.n.55 | e3668 | DOI: https://doi.org/10.25248/reas.e3668.2020 Página 7 de 9 
provém da educação é um processo contínuo que ocorre em qualquer momento, situação, mediante diferentes estratégias, desde que a pessoa esteja aberta para tal. O enfermeiro é visto como o profissional principal e responsável para ação do controle do câncer de mama através da realização de educação para as mulheres com orientações da importância da realização do autoexame das mamas e enfatizando a necessidade de observação de sinais de alterações possíveis nas mamas. Podemos concluir que o enfermeiro ainda apresenta dificuldades par exercer sua função sendo necessário preparo através de capacitações e conscientização da importância do mesmo para a detecção precoce do câncer de mama.

\section{REFERÊNCIAS}

1. AMERICAN CANCER SOCIETY. How common is breast cancer? Atlanta, 2020. Disponível em: https://www.cancer.org/cancer/breast-cancer/about/how-common-is-breast-cancer.html. Acesso em: 1 jan. 2020.

2. ANDRADE SAF. A importância do autoexame e exame clínico das mamas. Revista UNILUS Ensino e Pesquisa, 2014; 23(11): 112.

3. ASSOCIAÇÃO NACIONAL DE HOSPITAIS PRIVADOS. OMS divulga projeção de câncer mundial até 2040, dados não eram atualizados há 6 anos. São Paulo, 2018. Disponível em: https://www.anahp.com.br/noticias/noticias-domercado/oms-divulga-projecao-de-cancer-mundial-ate-2040-dados-nao-eram-atualizados-ha-6-anos. Acesso em: 4 fev. 2020.

4. AVELAR MP, et al. O papel da enfermagem no câncer de mama. Webartigos, 2014. Disponível em: https://www.webartigos.com/artigos/o-papel-da-enfermagem-no-cancer-de-mama/127116. Acesso em: 10 abr. 2020.

5. BRASIL. Ministério da Saúde. Câncer: sintomas, causas, tipos e tratamentos. Brasília, 2013.

6. BRASIL. Ministério da Saúde. Câncer de mama: sintomas, tratamentos, causas e prevenção. Brasília, 2020.

7. BRASIL. Ministério da Saúde. Falando sobre câncer de mama: realizando o exame clínico de mama. Brasília, 2001.

8. BRASIL. Ministério da Saúde. Instituto Nacional de Câncer José Alencar Gomes da Silva. Estimativa 2020: incidência de câncer no Brasil. Rio de Janeiro, 2019.

9. CAVALCANTE SAM, et al. Ações do enfermeiro no rastreamento e diagnóstico do câncer de mama no Brasil. Revista Brasileira de Cancerologia, 2013; 59(3): 459-466.

10. CENTRO DE INTEGRAÇÃO EMPRESA-ESCOLA. Conheça a história e a importância do Outubro Rosa. Paraná, 2018. Disponível em: https://www.cieepr.org.br/conheca-a-historia-e-a-importancia-do-outubro-rosa. Acesso em: 9 mar. 2020.

11. CONSELHO REGIONAL DE ENFERMAGEM. A atuação da enfermagem ao combate ao câncer de mama. Brasília, 2018. Disponível em: https://www.coren-df.gov.br/site/a-atuacao-da-enfermagem-no-combate-ao-cancer-de-mama. Acesso em: 13 abr. 2020.

12. CUNHA AR, et al. O papel do enfermeiro na orientação, promoção e prevenção do câncer de mama. Revista Humano Ser - UNIFACEX, 2017/2018; 3(1): 160-173.

13. ENDRIGO J, TRALDI MC. Conciencia sobre prevención del cáncer de mama y práctica de autoexamen en usuários del sistema público de salud. REFACS 2017; 5(2): 209-220.

14. FURTADO SB, et al. Compreendendo sentimentos das enfermeiras acerca do câncer de mama. Revista Rene, 2009; 10(4): 45-51.

15. INSTITUTO NACIONAL DE CÂNCER JOSÉ ALENCAR GOMES DA SILVA. ABC do câncer: abordagens básicas para o controle do câncer. 5. ed. rev. Rio de Janeiro, 2019.

16. INSTITUTO NACIONAL DE CÂNCER JOSÉ ALENCAR GOMES DA SILVA. Câncer de mama. Rio de Janeiro, 2020.

17. INSTITUTO NACIONAL DE CÂNCER JOSÉ ALENCAR GOMES DA SILVA. Detecção precoce do câncer de mama. Rio de Janeiro, 2018.

18. INSTITUTO NACIONAL DE CÂNCER JOSÉ ALENCAR GOMES DA SILVA. Diretrizes para a detecção precoce do câncer de mama no Brasil. Rio de Janeiro, 2015.

19. INSTITUTO ONCOGUIA. Câncer de mama. São Paulo, 2017.

20. INSTITUTO ONCOGUIA. Estatísticas para câncer de mama. São Paulo, 2020a.

21. INSTITUTO ONCOGUIA. O que são cuidados paliativos? São Paulo, $2020 \mathrm{~b}$.

22. INUMARU LE, et al. Fatores de risco e de proteção para câncer de mama: uma revisão sistemática. Caderno Saúde Pública, 2011; 27(7): 1259-1270.

23. LEÃO MRC, et al. Cuidados de enfermagem nos níveis de prevenção da história natural do câncer de mama. Percurso Acadêmico, 2011; 1(2): 270-286.

24. MARQUES EF, et al. Indications for breast magnetic resonance imaging in an oncology reference center. Radiologia Brasileira, 2011; 44(6): 363-366. 
25. MELO FBB, et al. Actions of nurses in early detection of breast cancer. Revista Brasileira Enfermagem, 2017; 70(6): 1183-1193.

26. OHL ICB, et al. Public actions for control of breast cancer in Brazil: integrative review. Revista Brasileira Enfermagem, 2016; 69(4): 793-803.

27. OLIVEIRA MM, et al. Ações do enfermeiro na detecção precoce do câncer de mama em mulheres no Brasil: revisão integrativa. Revista Científica FacMais, 2017; 11(4): 28-49.

28. PEREIRA ACA, et al. Sistematização da assistência de enfermagem e o câncer de mama entre mulheres. Revista de Ciências da Saúde Nova Esperança, 2018; 16(1): 39-47.

29. PROLLA CMD, et al. Knowledge about breast cancer and hereditary breast cancer among nurses in a public hospital. Revista Latino-Americana de Enfermagem, 2015; 23(1): 90-97.

30. RODRIGUES FB, et al. O papel do enfermeiro na prevenção do câncer de mama em um município do sertão pernambucano: uma abordagem da prática profissional. Saúde Coletiva em Debate, 2012; 2(1), 73-86.

31. SILVA PA, RIUL SS. Câncer de mama: fatores de risco e detecção precoce. Revista Brasileira Enfermagem, 2011; 64(6): 1016-1021.

32. SOARES CB. Ações educativas para realização do autoexame de mamas. Trabalho de Conclusão de Curso (Graduação) - Escola de Enfermagem. Universidade Federal do Rio Grande do Sul, Porto Alegre, 2011; 51 p.

33. STEIN AT, et al. Rastreamento do câncer de mama: recomendações baseadas em evidências. Revista da AMRIGS, 2009 ;53(4): 438-446.

34. TEIXEIRA MS, et al. Atuação do enfermeiro da Atenção Primária no controle do câncer de mama. Acta Paulista Enfermagem, 2017; 30(1): 1-7.

35. VEIT MT, CARVALHO VA. Psico-oncologia: um novo olhar para o câncer. O Mundo da Saúde, 2010; 34(4): 526-530.

36. WORLD HEALTH ORGANIZATION. International Agency for Research on Cancer. Global Cancer Observatory. Geneva, 2020 . 